\title{
Need for community-based primary prevention of coronary heart disease in Nepal
}

Vaidya A

Department of Community Medicine, Kathmandu Medical College, Duwakot, Bhaktapur, Nepal

$\mathrm{C}$ oronary Heart Diseases (CHD) are the leading cause of death worldwide and most of these deaths occur in the developing countries ${ }^{1}$. The current evidences indicate that deaths due to CHD are decreasing in developed countries. On the other hand, with ageing populations and rapidly changing lifestyles, the burden of CHD is increasing in almost all developing countries including Nepal. Apart from the hospital-based data that show a steady rise in the number of patients suffering from CHD, community-based studies in Nepal have also indicated a high prevalence of the disease and its risk factors in the general population. For example, a pioneering study in Dharan found that almost six percent of the adult males had an evidence of $\mathrm{CHD}^{2}$. The underlying risk factors were also very high in the population: about $7 \%$ were diagnosed cases of diabetes mellitus, nearly $25 \%$ had hypertension, $40 \%$ were overweight, $45 \%$ had inadequate physical activity, 50\% had increased waist-to-hip ratio, and $75 \%$ were either current or past users of tobacco ${ }^{3-4}$.

The concept of community intervention was introduced in developed countries in the late 1960s and the current decline in the CHD in these countries has been attributed to the long term promotion of healthy lifestyles and community prevention measures. Some excellent examples of community-based projects for prevention of CHD in Europe and United States of America include: the North Karelia Project (Finland), Stanford Five-City Project (USA), the German Cardiovascular Prevention Study, Minnesota Heart Health Programme (USA), the Swiss National Research Programme, and the Kilkenny Health Project (Ireland). Among them, North Karelia Project is the most successful one, which was first conducted as demonstration programme, and after the original project period, their experience has actively been applied for national action ${ }^{5}$.

The primary interventions can either be targeted towards the individuals with one or more high risks such as high cholesterol, etc (called High-Risk Approach) or towards the whole population who will have a range of various risk factors (called Population Approach), or a combination of both. While the High-Risk Approach attempts to modify the abnormal risk factor profile of an individual, the Population Strategy aims to bring about behavioral changes and lifestyle modifications in the whole population through innovative media campaigns, community participation and co-operation with local and national sectors and policy making.

The results of programmes using high-risk approach demonstrated that they could reduce the incidence of cardiovascular diseases among the high risk individuals even in less than five years. However, most of the cardiovascular diseases occur in the people not at the highest risk level but in those who are at high-normal to borderline level, simply because there are more people in the latter group. So, the high-risk approach will do little to bring down the number of cases of cardiovascular diseases, especially in the developing countries. Because of this fact, most of the large-scale community-based cardiovascular disease intervention projects use population approach or combination of high-risk approach and population approach called as Comprehensive Community-based Approach. Though the changes in risk achieved in population-based studies are often small, even small changes in the distribution of risk factors in the population will bring about sizable changes in cardiovascular mortality ${ }^{6}$.

Many prevention programmes have targeted only one risk factor of cardiovascular diseases in order to test the effect of the targeted risk factor change and its impact on cardiovascular disease incidence, morbidity or mortality. Such single risk management programmes include, for example, high blood pressure control, cholesterol concentration reduction, changes in nutrition, and community-based smoking cessation. Although such an approach can be effective, many studies have demonstrated that, to achieve the greatest benefits, a paradigm shift is required from the treatment of risk factors in isolation to a comprehensive cardiovascular risk-management approach. The reason for focus on integrated multi-factorial approach is also that it is very common for two or more cardiovascular risk factors to occur together in one person, and the clustering of risk factors may act synergistically increasing the risk 
more than any one single factor acting alone. Hence, Community-based primary prevention of CHD should target main cardiovascular risk factors, especially four behavioral risk factors (tobacco, unhealthy diet, physical inactivity, alcohol) using comprehensive riskmanagement strategies.

The successful experiences and scientific evidence for $\mathrm{CHD}$ prevention achieved in the past 40 years in developed countries make it possible for the developing countries to find the appropriate approaches to address these diseases ${ }^{7}$. Like any developing country, Nepal is challenged by poverty, communicable diseases, high maternal deaths, and malnutrition and the problem of non-communicable diseases such as cardiovascular diseases has remained largely ignored. Due to lack of proper policies and programmes on its prevention, CHD and its risk factors have silently fostered in the background and are now rapidly emerging as an important public health problem. Even now, most of the attention and resources are being disproportionately channelised towards the curative aspect of the disease while the prevention component has remained limited to some awareness programmes run by local clubs, sporadic camps or celebrations of World Heart Day, etc. This is critical time to pay heed to preventive programmes in cardiovascular disease. For this, prevention at the community-level is essential because modifiable causal risk factors are deeply entrenched in the social and cultural framework of our society. As said earlier, the evidence indicates that community-based primary prevention is needed for cardiovascular disease prevention and control. Let us act now before it is too late!

\section{References}

1. Murray CJL, Lopez AD. Mortality by cause for eight regions of the world: Global Burden of Disease Study. Lancet 1997;349:1269-76.

2. Vaidya A, Pokharel PK, Nagesh S, Karki P, Kumar S, Majhi S. War veterans of Nepal and their blood pressure status: a populationbased comparative study. Journal of Human Hypertension 2007;21:900-3.

3. Vaidya A, Pokharel PK, Karki P, Nagesh S. Exploring the iceberg of hypertension: A community based study in an Eastern Nepal town. KUMJ 2007;5(3):349-59.

4. Vaidya A, Pokharel PK, Nagesh S, Karki P, Kumar S, Majhi S. Association of Obesity and Physical Activity in Adult Males of Dharan, Nepal. KUMJ 2006;4(2):192-7.

5. Puska P. Successful Prevention of Noncommunicable Diseases: 25 year experiences with North Karelia Project in Finland. Public Health Medicine 2002;4(1):5-7.

6. Shea S, Basch CE. A review of five major community-based cardiovascular disease prevention programmes. Part I: Rationale, design, and theoretical framework. American Journal of Health Promotion 1990;4(3):203-13.

7. Nissinen A, Berrios X, Puska P. Communitybased Non communicable Disease Interventions: lessons from developed countries for developing ones. Bulletin of the World Health Organization 2001;79:963-70. 